\title{
Incision Site
}

National Cancer Institute

\section{Source}

National Cancer Institute. Incision Site. NCI Thesaurus. Code C77683.

The anatomic site of a cut made during surgery. The term may also refer to the resultant scar from the surgical procedure. 